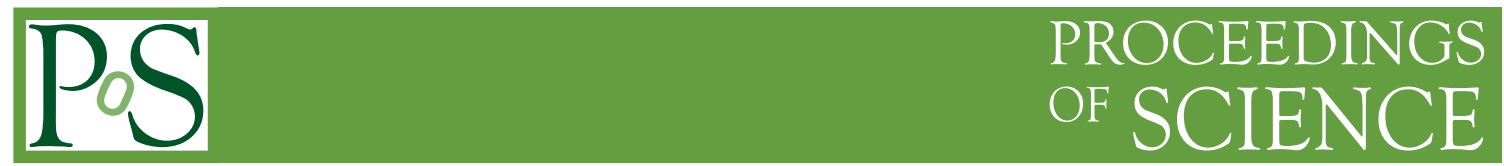

\title{
Inhomogeneous and Quarkyonic Phases of High Density QCD
}

\section{Larry McLerran*}

Physics Department and RIKEN BNL Brookhaven National Laboratory, Upton, NY 11973;

Physics Department, China Central Normal University, Wuhan, China

\begin{abstract}
I review the properties of Quarkyonic Matter. Based on large numbers of colors arguments, this matter may exist at very high baryon number density. Quarkyonic Matter is confining even when the quark chemical potentials are large compared to $200 \mathrm{MeV}$. It has broken chiral symmetry with non-translationally invariant chiral condensates. There may be an isolated region in the QCD phase diagram corresponding to such matter.
\end{abstract}

9th International Workshop on Critical Point and Onset of Deconfinement - CPOD2014,

17-21 November 2014

ZiF (Center of Interdisciplinary Research), University of Bielefeld, Germany

${ }^{*}$ Speaker. 


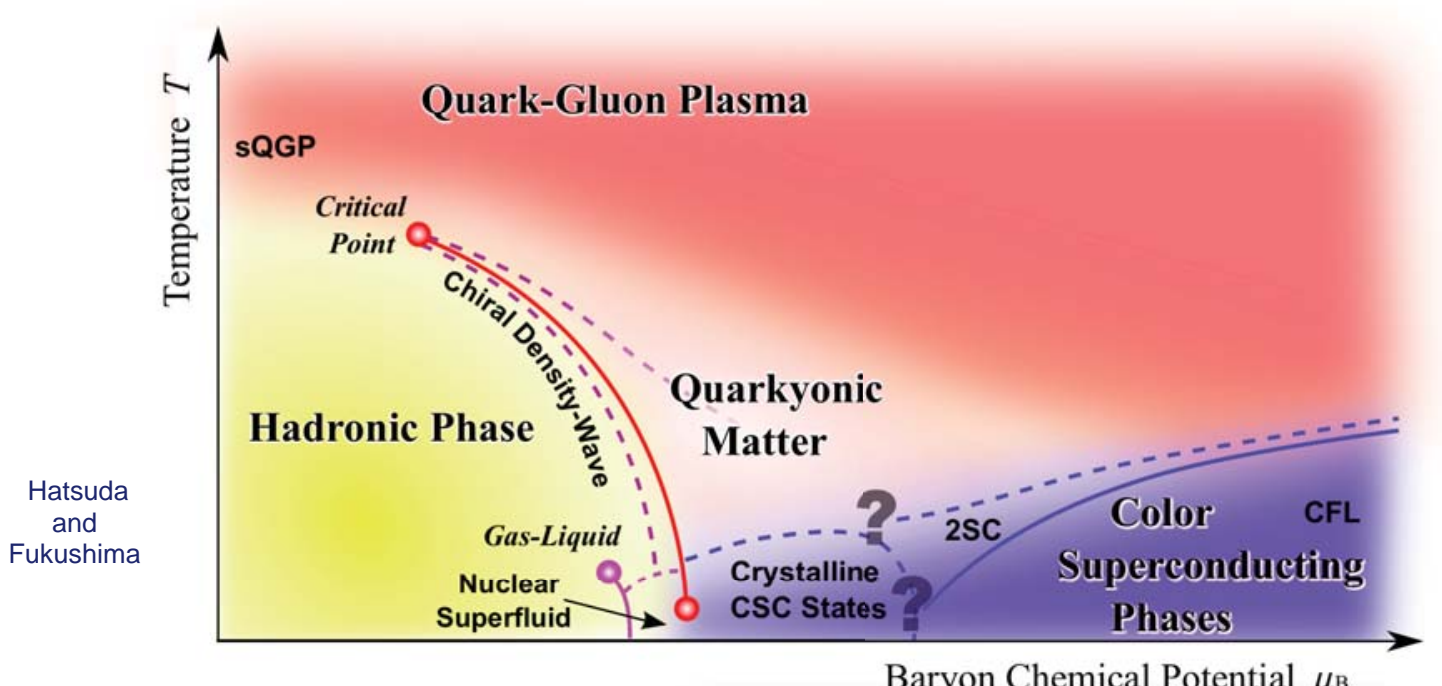

Figure 1: A hypothetical phase diagram of QCD at finite temperature and baryon chemical potential[1].

\section{Introduction}

The phase diagram of QCD as currently envisioned has many possibilities for new phases of matter[1]. At low temperature and density matter is a gas of hadrons. At high temperature and low baryon density it is a Quark Gluon Plasma. At the highest baryon densities and not too high temperature, it is a Color Superconductor[2]-[3]. At high baryon densitiy and temperautres, it has been recently proposed that there is matter called Quarkyonic Matter[4]-[5].

The possible existence of Quarkyonic Matter may be inferred from arguments based on the approximation that the number of colors $N_{c} \rightarrow \infty$. In this limit the number of gluons is large $N_{g l}=2\left(N_{c}^{2}-1\right) \rightarrow \infty$, and the ratio of the number of quarks to gluons $2 N_{c} N_{f} /\left(N_{c}^{2}-1\right) \rightarrow 0$. In perturbation theory, one introduces the 't Hooft coupling, $\alpha^{\prime}{ }_{t H o o f t}=\alpha_{S} N_{c}$ which is held finite as $N_{c} \rightarrow \infty$. The effects of gluon loops and quantum corrections are finite in this limit, but the effect of quark loops is small, $\sim 1 / N_{c}$.

The masses of mesons are finite in the large $N_{c}$ limit, but baryons are very massive $M_{B} \sim$ $N_{c} \Lambda_{Q C D}$, since baryons contain $N_{c}$ quarks. Therefore, in ordinary hadronic matter there are no baryons nor effects of baryons in quantum corrections. In the Quark Gluon Plasma, there are baryons in the form of deconfined quarks, since the mass of a single quark is finite in the large $N_{c}$ limit. If one makes the baryon chemical potential larger than the nucleon mass, which at zero temperature is the threshold chemical pontential for generating a Fermi sea of baryons, then there can be a finite baryon density. The quantity $e^{\left(\mu_{B}-M_{B}\right) / T}$ is an order parameter in the large $N_{c}$ limit for baryon number density. We will call matter at high baryon density and lowish temperature $T \leq \Lambda_{Q C D}$ Quarkyonic Matter.

The confining properties of QCD also differentiates between Hadronic Matter, the Quark Gluon Plasma, and Quarkyonic Matter. One imagines the disappearence of confinement at finite temperature as due to Debye screening. This arises from a 1-loop correction to the gluon propagator from finite temperature gluons. This gives a Debye mass $M_{D}^{2} \sim \alpha N_{c} T^{2}$. Since $\alpha_{S} N_{c}$ is finite in 
the large $N_{c}$ limit, there can exist a finite temperature phase of gluons where the confining potential is screened. At high baryon density and low temperature, the Debye mass is of order $M_{D}^{2} \sim \alpha_{S} \mu_{Q}^{2}$, where $\mu_{Q}$ is the quark chemical potential, $\mu_{Q}=\mu_{B} / / N_{c}$. The Debye mass for quarks will not affect the confining properties of the theory so long as $\mu_{Q}<<\sqrt{N_{c}} \Lambda_{Q C D}$. This is in a region where it is possible that $\mu_{Q}>>\Lambda_{Q C D}$, where the coupling evaluated at this scale is weak.

How is it possible that the weakly coupled theory at finite density can be confining? The solution is that perturbation theory breaks down for quarks near the Fermi surface. Deep inside the Fermi sea, quarks must scatter with large momentum, since scattering must involve promoting a quark inside the Fermi sea to an energy above that of the Fermi energy. For large quark chemical potential, this involves a big energy compared to the QCD scale, and will be controlled by a weak coupling. For quarks near the Fermi sea, there is no such restriction, and there can be processes which are non-perturbative and involve strong coupling.

The picture that arises from these considerations is amusing: Inside the Fermi sea there are deconfined quarks. At the Fermi surface, quarks are confined into baryons. The thermal excitations of this system are mesons and glueballs. This dual feature of high density matter leads to the word Quarkyonic, which is a word half made of quark and the last half of baryonic.

The energy density of hadronic matter is of order one in the large $N_{c}$ limit. When one makes a transition to a Quark Gluon Plasma, the number of degrees of freedom changes by of order $N_{c}^{2}-1$, corresponding to the number of colors of gluons, and the energy density is of this order. In Quarkyonic Matter, gluons are confined, so the number degrees of freedom and the energy density are of order $N_{c}$, corresponding to the number of colors of quarks. Therefore, in the large $N_{c}$ limit, the energy density of matter is an order parameter for the transitions between a Hadron Gas, the Quark Gluon Plasma and Quarkyonic Matter.

The dynamics that induces the finite temperature QCD phase transition may be understood in the language of hadron resonances. In large $N_{c}$, there is an infinite spectrum of hadron resonances. The interaction strength of these resonances may be argued to be of order $1 / N_{c}$. There is therefore an accumulation of resonances until some very high density of resonances where the interactions become important. Eventually the interactions are so strong that the quarks and gluons that make the resonances are freed, and one makes a Quark Gluon Plasma.

At finite density and low temperature, for quark chemical potential less han the nucleon mass there are no nucleons present. At zero temperatures, at a chemical potential $\mu_{Q}=M_{B} / N_{c}+$ $O\left(\Lambda_{Q C D} / N_{c}\right)$, the baryons appear. The baryons once they appear are very strongly interacting, and form Quarkyonic Matter.

\section{Chiral Symmetry Restoration}

Chiral symmetry if unbroken requires either massless baryons and parity doubled hadrons with finite mass. The hadronic world is a world of broken chiral symmetry. If one pairs a $0^{++} \sigma$ meson with the $0^{+-}$triplet of pions, there is a realization of chiral symmetry as an $O(4)$ rotational symmetry associated with these particles. Chiral symmetry is broken by a condensation of $\sigma$ mesons. With this breaking, the pions are Goldstone bosons, which would be massless in the massless quark limit corresponding to exact chiral symmetry. 
At finite temperature, there is some temperature associated with the restoration of chiral symmetry. At this temperature, the quarks lose their constituent masses and are to a good approximation massless.

At finite density, the dynamics is complicated by the Fermi surface. Near the Fermi surface, quarks are bound into baryons and the excitations of the Fermi sea are mesons. If we try to form a chiral condensate, it would be made of a particle hole pairs from the Fermi sea. The energy of each of the quark and the quark-hole is of order the Fermi energy. To bind together, the quark and quarkhole pair must have small relative momentum. If the meson has a net energy $2 \mu_{Q}$, then it must have net momentum. Therefore the chiral condensate will be made of moving particles. There will be a DeBroglie wavelength associated with this condensate $\lambda=1 / \mu_{Q}$ so we expect the condensate will break translational invriance. The condensation is similar to the formation of charge-density waves in condensed matter physics.

The existience of such inhomgeneous chiral condensates has now been established in a number of models of Hadronic Matter[6]-[9]. The pheonomenon is quite robust. If it occurs, then Quarkyonic Matter will be a region surrounded by a line of phase transitions associated with the breaking of translational invariance.

The nature of the Quarkyonic phase can be quite complicated. It is possible to solve the sigma model at high densitiies for matter near a Fermi surface of high density matter[10]. This is valid so long as one does not probe degrees of freedom at a momentum scale much greater than the QCD scale as a distance in momentum from the Fermi surface. As Quarkyonic Matter appears, it spontaneously breaks chiral symmetry by a condensate which is not tranlsationally and rotationally invariant. The sigma model tries to reestablish rotational invartiance by making a crystal of condensate domains oriented in different directions in momentum space. In 2+1 dimensions, one finds at low densities a square lattice structure which transforms into higher order polygonal structures as the density increases. One can attain a $N_{c}$ order polygonal structure if one increases the density. In coordinate space, one in general finds lattices which are not strictly periodic, and are quasi-crystals.

\section{A Simple Picture of the Phase Boundary}

The finite temperature transition in large $N_{c}$ occurs at a temperature independent of $N_{c}$ as $N_{c} \rightarrow \infty$. In the $\mu_{B}-T$ plane it is a straight line at $T=T_{c}$. The Quarkyonic transition should occur when $e^{\left(\mu_{B}-M_{n}\right) / T} \sim 1$, or $\mu_{B}=M_{n}-c T$ where $c$ is a constant of order 1 . In the $\mu_{B}-T$ plane, the phase boundary between Quarkyonic Matter and Hadronic Matter is a straight line intersecting the $\mu_{B}$ axis at $M_{N}$ when the temperature shrinks to zero.

In Figure 2, a plot of the phase bundary is shown[11]. For comparison, the chemical potentials and temperatures of decoupling in various heavy ion experiments is shown. The decoupling might arise due to the large change in the energy density scales on the boundaries between the various phases. Note that there is triple point where the three phases of Quarkyonic Matter, the Quark Gluon Plasma and Quarkyonic Matter can coexist. This might be a place where a critical end point might occurs. It might also occur to the left of the triple point.

A test of the large $N_{c}$ limit is how constant is the decoupling temperature in the RHIC data[12]. This is shown in Fig. 3, and indeed at low chemical potnetial it appears that the large $N_{c}$ arguments agree well with the data[12]. 


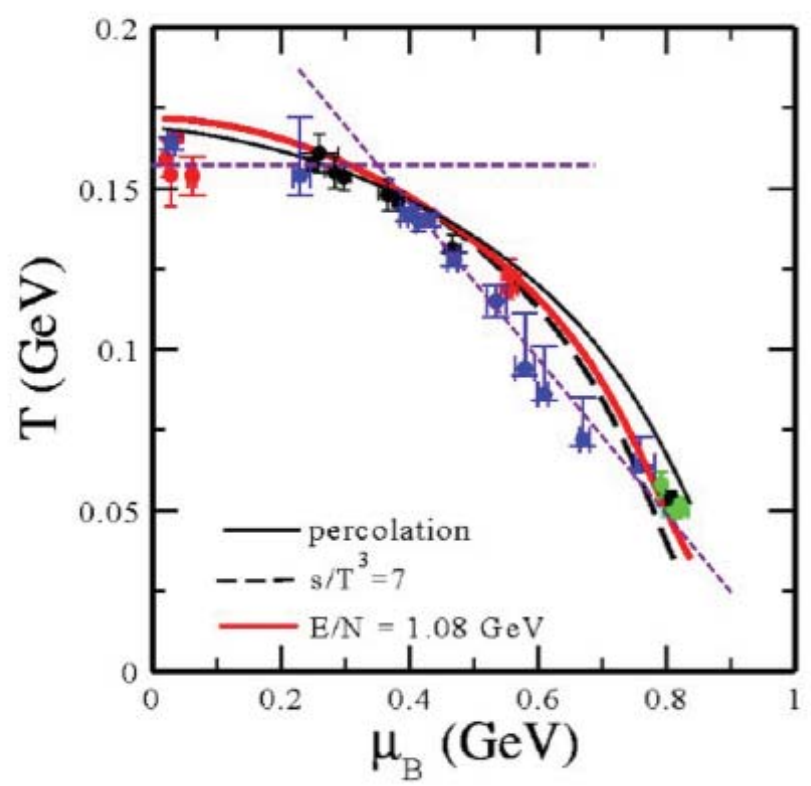

Figure 2: Comparison of a very simple model of the phase boundaries between Hadronic Matter, the Quark Gluon Plasma and Quarkyonic matter and data on the temperature and density of decouplingl[11].

\begin{tabular}{|c|c|c|c|c|c|}
\hline is (GeV) & $\begin{array}{c}\text { Statistics(Millions) } \\
(0-80 \%)\end{array}$ & Year & $\mu_{\mathrm{s}}$ (MeV) & T (MeV) & $\mu_{\mathbf{g}} \pi$ \\
\hline 7.7 & $\sim 3$ & 2010 & 422 & 140 & 3.020 \\
\hline 11.5 & $\sim 6.6$ & 2010 & 316 & 152 & 2.084 \\
\hline 19.6 & $\sim 15$ & 2011 & 206 & 160 & 1.287 \\
\hline 27 & $\sim 32$ & 2011 & 156 & 163 & 0.961 \\
\hline 39 & $\sim 86$ & 2010 & 112 & 164 & 0.684 \\
\hline 62.4 & $\sim 45$ & 2010 & 73 & 165 & 0.439 \\
\hline 200 & $\sim 238$ & 2010 & 24 & 166 & 0.142 \\
\hline
\end{tabular}

Figure 3: A computation from STAR data of decoupling temperatures at low baryon number density [12].

To understand the isolated region where one has Quarkyonic Matter, a variety of computations in various models have been performed[7]-[9]. The results of some of these computations are shown in Fig.4. The existence of an isolated region corresponding to Quarkyonic Matter is robust through many different computations, although there is not consensus about what is the correct model, nor on the details of the mechanism which generates an inhomogenous chiral condensate.

\section{Phenomenological Implications}

If one assumes the decoupling surface is close to the lines associated with the phase transitions to the Quark Gluon Plasma and to Quarkyonic matter, there are consequences. Gadzicki and Gorentsein long ago observed non-monotnic behaviour of various particle ratios as a function of 


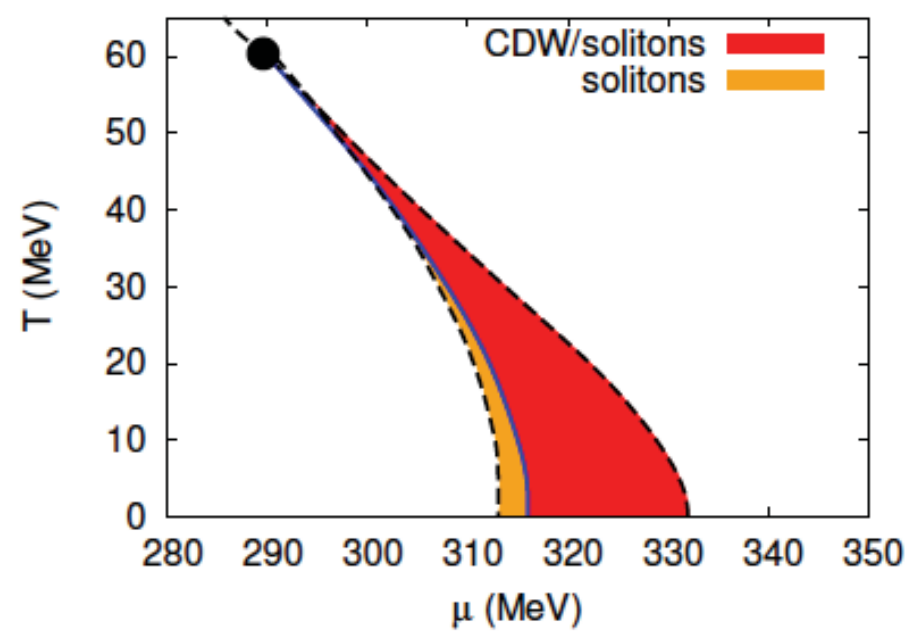

Figure 4: The region in the $\mu_{Q}-T$ plane where there is an inhomogenous chiral condensate. [7]-[9].

quark chemical potential and density[13]. For example, one can follow the decoupling curve as a function of beam energy. This is shown for various particle ratios in Fig. 5. The non-monotonic behvaviour occurs when one goes from the Quarkyonic branch of the deoupling curve to the QGP branch. This occurs close to the region where these two branches meet, as seen in Fig. 2

One can also check to see whether one is going from baryonic rich matter on the Quarkyonic branch of the curve to meson rich matter on the Quark Gluon Plasma branch. As shown in Fig. 6, this appears to be the case.

\section{Summary and Conclusions}

In fact, little is known about the properties of matter at lowish temperature and high baryon number density. Quarkyonic Matter provides some crude idea of what matter might be like.. The ideas discussed in this paper are imaginative, but perhaps not imaginative enough. At present, lattice Monte-Carlo methods do not work for such a region, and there is little rigorous theoretical knowledge. Also, phenomenological tools needed to make sense of theoretical results are poorly developed for the energy range of heavy ion collisions which might provide an opportunity to study such matter. There is therefore a great opportunity for discovery, both in experiment and theory.

Among the theoretical issues that are important is to better understand how inhomogeneous chiral condensates manifest themselves. We also need a better understanding of where the critical end point is relative to the triple point. We need to have much better control of computations of heavy ion collisions at the energy range where such matter might be produced and studied. Currently, the models used are not at the same level of credibility as are the models for the high temperature baryon free region. 


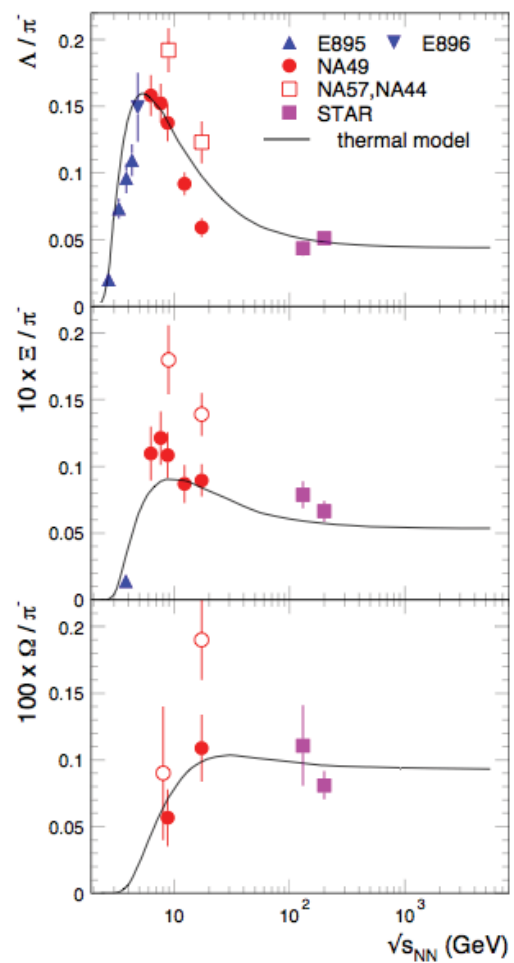

Figure 5: Various particle ratios as a function of colliding beam energy [13].

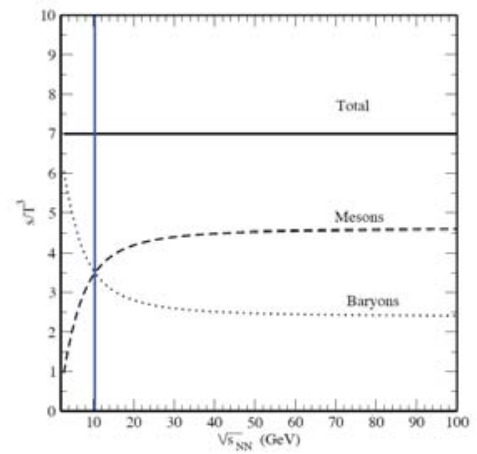

Figure 6: Baryon versus meson contributions at decoupling [11].

\section{Acknowledgments}

This research was supported by the Office of Science, Department of Energy contract number Contract No. DE-SC0012704. I greatly acknowledge also support as a Hans Jensen Professor at the Theoretical Physics Institute of the University of Heidelberg. 


\section{References}

[1] K. Fukushima and T. Hatsuda, Rept. Prog. Phys. 74, 014001 (2011) [arXiv:1005.4814 [hep-ph]].

[2] M. G. Alford, K. Rajagopal and F. Wilczek, Phys. Lett. B 422 (1998) 247 [hep-ph/9711395].

[3] R. Rapp, T. SchÃd'fer, E. V. Shuryak and M. Velkovsky, Phys. Rev. Lett. 81 (1998) 53 [hep-ph/9711396].

[4] L. McLerran and R. D. Pisarski, Nucl. Phys. A 796, 83 (2007) [arXiv:0706.2191 [hep-ph]].

[5] Y. Hidaka, L. D. McLerran and R. D. Pisarski, Nucl. Phys. A 808, 117 (2008) [arXiv:0803.0279 [hep-ph]].

[6] T. Kojo, Y. Hidaka, L. McLerran and R. D. Pisarski, Nucl. Phys. A 843 (2010) 37 [arXiv:0912.3800 [hep-ph]].

[7] M. Buballa and S. Carignano, Phys. Rev. D 87 (2013) 5, 054004 [arXiv:1210.7155 [hep-ph]].

[8] S. Carignano, M. Buballa and B. J. Schaefer, Phys. Rev. D 90 (2014) 1, 014033 [arXiv:1404.0057 [hep-ph]].

[9] M. Buballa and S. Carignano, arXiv:1406.1367 [hep-ph].

[10] T. Kojo, Y. Hidaka, K. Fukushima, L. D. McLerran and R. D. Pisarski, Nucl. Phys. A 875 (2012) 94 [arXiv:1107.2124 [hep-ph]].

[11] A. Andronic, D. Blaschke, P. Braun-Munzinger, J. Cleymans, K. Fukushima, L. D. McLerran, H. Oeschler and R. D. Pisarski et al., Nucl. Phys. A 837 (2010) 65 [arXiv:0911.4806 [hep-ph]].

[12] Presentation by STAR at this meeting.

[13] M. Gazdzicki and M. I. Gorenstein, Acta Phys. Polon. B 30 (1999) 2705 [hep-ph/9803462]. 